\title{
Near-exact explicit asymptotic solution of the SIR model well above the epidemic threshold
}

\author{
Gregory Kozyreff \\ Physics Department, Université libre de Bruxelles (U.L.B.), CP 231, \\ Campus de la Plaine, 1050 Bruxelles, Belgium.
}

March 24, 2021

\begin{abstract}
A simple and explicit expression of the solution of the SIR epidemiological model of Kermack and McKendrick is constructed in the asymptotic limit of large basic reproduction numbers $\mathrm{R}_{0}$. The proposed formula yields good qualitative agreement already when $R_{0} \geq 3$ and rapidly becomes quantitatively accurate as larger values of $R_{0}$ are assumed. The derivation is based on the method of matched asymptotic expansions, which exploits the fact that the exponential growing phase and the eventual recession of the outbreak occur on distinct time scales. From the newly derived solution, an analytical estimate of the time separating the first inflexion point of the epidemic curve from the peak of infections is given.
\end{abstract}

\section{Introduction}

The COVID-19 pandemic has impacted all aspects of our daily lives. It has thus triggered an extraordinary world-wide response across all fields of science, from virology and pharmacology to sociology. Within the scientific crowd, applied mathematicians and theoretical physicists also brought their contribution, notably by using their mathematical expertise in epidemiological modelling $[1,2,3,4,5,6,7,8,9,10,11,12]$. Deterministic compartment models allow one in principle to develop an accurate global view of the contagion dynamics within large populations. Compartments can be used to divide the population into age categories as well as according to the evolution of the illness, for those who have been infected $[13,8,10]$. These multiple levels of description are useful and necessary, and can be made to fit closely to the data. However, they contain a plethora of fitting parameters and can therefore be complex to interpret. Fortunately, it turns out that the simplest of all compartment models, SIR, faithfully reproduces the global dynamics at the level of a country or a large city with COVID-19 $[9,14]$. As a result, the SIR model remains a useful 
tool at such global level of description. We write it as follows

$$
\begin{aligned}
\frac{\mathrm{d} S}{\mathrm{~d} t} & =-\beta S I / N, \\
\frac{\mathrm{d} I}{\mathrm{~d} t} & =\beta S I / N-\gamma I, \\
\frac{\mathrm{d} R}{\mathrm{~d} t} & =\gamma I,
\end{aligned}
$$

where $S, I$, and $R$ respectively denote the number of susceptible, infected and removed individuals, with constant sum $N=S+I+R$. The population $R$ includes both those who have recovered from the illness and those who have died. Above, $\beta$ is the contact rate, $\gamma$ is the recovery rate, and their ratio

$$
\mathrm{R}_{0}=\beta / \gamma
$$

is the basic reproduction number. Despite its long history [15], there continues to be many efforts to try and solve it analytically $[16,17,18,19,20,21,22,23,24$, 25]. In their seminal paper, Kermack and McKendrick offered an approximate solution in the limit of a "small epidemic", i.e. when the reproduction number $R_{0}$ is just above 1 [15]. So far, this has remained the only explicit approximate analytical solution available, in the sense that it can be justified as an asymptotic limit of the true solution. Well above the epidemic threshold, an analytical expression of the solution can be written in parametric form [26]. Unfortunately, it is only given implicitly, such that time is expressed in terms of one of the dynamical variables through an integral. Hence, this solution is not directly interpretable and its analysis can be quite involved [23]. Alternatives have been proposed in the form of converging series [17, 20], but these can involve expansions with as many as 15 up to 60 terms and, hence, are again impractical for analysis. More down-to-earth is the approach by which the solution is fitted by well-chosen ansatz with a few parameters [27, 24]. However, these ansatz are not derived from the model and their parameters must be fitted to the data, rather than deduced from the model.

\section{$1.1 \quad$ Statement of results}

The aim of this paper is to show that

$$
\frac{R(t)}{N} \equiv n_{r}(t) \sim\left\{\begin{array}{cl}
\frac{1}{\mathrm{R}_{0}-1} \ln \left[a e^{(\beta-\gamma) t}+1-a\right], & t<t_{*}, \\
\frac{1}{\mathrm{R}_{0}-1} \ln \left[1+\frac{1-a}{a} e^{-(\beta-\gamma) t}\right]+n_{r, \infty}-e^{-\gamma\left(t-t_{*}\right)}, & t>t_{*},
\end{array}\right.
$$

where $a$ is the infected fraction of the population at $t=0$, while $n_{r, \infty}$ and $t_{*}$ are given by

$$
n_{r, \infty} \sim 1-e^{-\mathrm{R}_{0}\left(1-e^{-\mathrm{R}_{0}}\right)}, \quad \quad t_{*} \sim \frac{n_{r, \infty}-1}{\gamma}-\frac{\ln a}{\beta-\gamma} .
$$

Given $n_{r}(t)$, one may infer the infected fraction of the population exactly as

$$
\frac{I(t)}{N} \equiv n_{i}(t)=1-n_{r}(t)-(1-a) e^{-\mathrm{R}_{0} n_{r}(t)}
$$



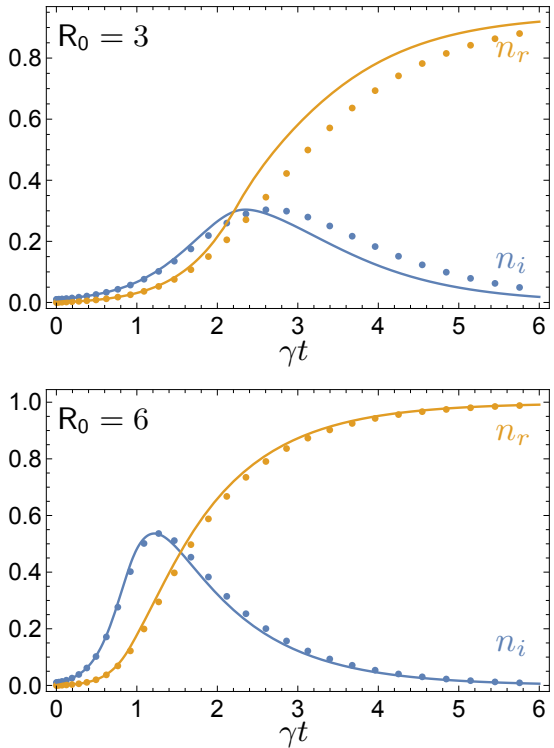
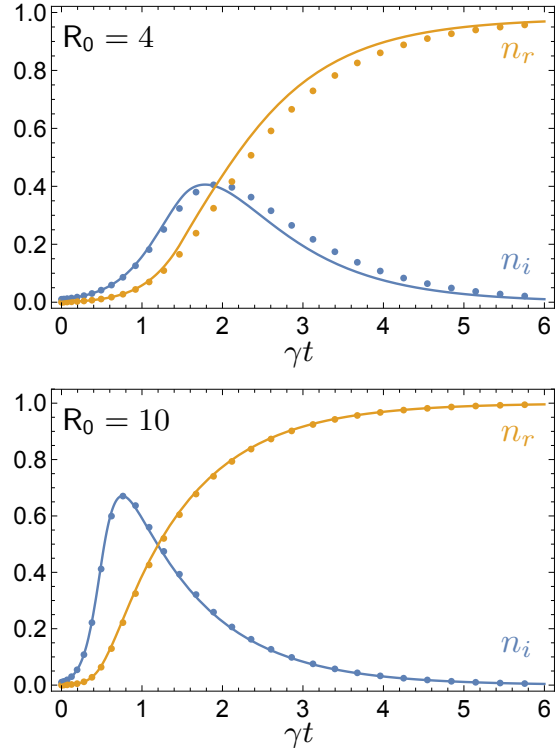

Figure 1: Comparison between the approximate formulas (1) and (3) (full lines) and the numerical solution of the SIR equation (dotted lines) for several values of the reproduction number $\mathrm{R}_{0} \cdot n_{i}(0)=a=0.01$.

Eq. (1) is remarkably simple and compact. The " $\sim$ " sign in it indicates that it holds asymptotically in the limit $\mathrm{R}_{0} \gg 1$. However, a good qualitative agreement with the numerical solution is already found for $\mathrm{R}_{0}=3$ and the approximate formula rapidly becomes as good as exact with increasing $\mathrm{R}_{0}$, see Fig. 1 . Hence, Eq. (1) complements the classical "small epidemic" formula derived by Kermack and McKendrick [15], which is valid in the limit $0<\mathrm{R}_{0}-1 \ll 1$. When, in 1956 , D. G. Kendall presented the implicit analytical solution of the SIR model, he noted:

"It is curious that the Kermack and McKendrick approximation should have been accepted without comment for nearly thirty years; the exact solution is easily obtained and the difference between the two can be of practical significance."

Similarly, the reader will find that the derivation of the present asymptotic formula is quite simple and could have been established much earlier with wellestablished asymptotic techniques.

As an illustration of the usefulness of the present analytical expression, let us compute the time elapsed between two important moments of the epidemic, namely the time $t_{1}$ of first inflexion of the curve $n_{i}(t)$ and the time $t_{2}$ of the peak of the epidemic. In appendix, we show that the former happens when $n_{r}$ is equal to $n_{1}$, solution of

$$
\mathrm{R}_{0} \frac{\mathrm{R}_{0} e^{-\mathrm{R}_{0} n_{1}}}{1-\mathrm{R}_{0} e^{-\mathrm{R}_{0} n_{1}}}=\frac{1-\mathrm{R}_{0} e^{-\mathrm{R}_{0} n_{1}}}{1-n_{1}-e^{-\mathrm{R}_{0} n_{1}}},
$$




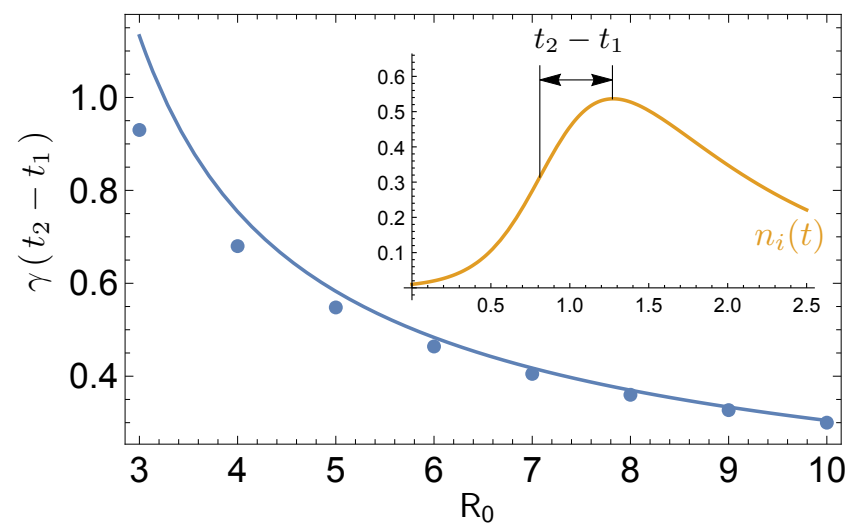

Figure 2: Time from inflexion to epidemic maximum as a function of $\mathrm{R}_{0}$. Dots: numerical solution. Full line: asymptotic curve, Eq. (9).

while the latter happens when

$$
n_{r}=n_{2}=\frac{\ln \mathrm{R}_{0}}{\mathrm{R}_{0}}
$$

in the limit $a \rightarrow 0$. It turns our that $t_{1}<t_{*}$ and that $t_{2}>t_{*}$. Hence, using Eq. (1), we have

$$
\gamma t_{1} \sim \frac{\ln (1 / a)}{\mathrm{R}_{0}-1}+\frac{1}{\mathrm{R}_{0}-1} \ln \left[e^{\left(\mathrm{R}_{0}-1\right) n_{1}}-1\right] .
$$

Meanwhile, for $t_{2}$, we have

$$
n_{2}=\frac{1}{\mathrm{R}_{0}-1} \ln \left[1+\frac{1-a}{a} e^{-(\beta-\gamma) t_{2}}\right]+n_{r, \infty}-e^{-\gamma\left(t_{2}-t_{*}\right)} .
$$

Above, we may assume in first approximation that $t_{2}$ is sufficiently large that the logarithmic term is negligible. Thus, we obtain the more manageable expression

$$
n_{2} \sim n_{r, \infty}-e^{-\gamma\left(t_{2}-t_{*}\right)}, \quad \rightarrow \gamma t_{2} \sim \gamma t_{*}+\ln \left(n_{r, \infty}-n_{2}\right),
$$

and obtain

$$
\gamma\left(t_{2}-t_{1}\right) \sim n_{r, \infty}-1+\ln \left(n_{r, \infty}-n_{2}\right)-\frac{1}{\mathrm{R}_{0}-1} \ln \left[e^{\left(\mathrm{R}_{0}-1\right) n_{1}}-1\right] .
$$

A comparison between this approximate formula and the numerics is given in Fig. 2. Collecting data in real-time allows one to detect when the slope of the epidemic curve has reached a maximum; $t_{2}-t_{1}$ is then the time left before the epidemic starts to recede.

\section{Preliminaries}

Before embarking into the asymptotic analysis of the SIR model, we first recall a few well-known facts. Eliminating $S$ thanks to the relation $S(t)+I(t)+R(t)=$ 
$N$, the evolution equations for the fractions $n_{i}=I / N$ and $n_{r}=R / N$ are

$$
\begin{aligned}
\frac{\mathrm{d} n_{i}}{\mathrm{~d} t} & =(\beta-\gamma) n_{i}-\beta\left(n_{i}+n_{r}\right) n_{i}, \\
\frac{\mathrm{d} n_{r}}{\mathrm{~d} t} & =\gamma n_{i} .
\end{aligned}
$$

They should be solved with the initial condition

$$
n_{i}(0)=a \ll 1, \quad n_{r}(0)=0 .
$$

At the start of the outbreak, $n_{i}, n_{r} \ll 1$ and the linearised evolution equations immediately yield

$$
n_{i} \approx a e^{(\beta-\gamma) t}, \quad n_{r} \approx \frac{a}{\mathrm{R}_{0}-1} e^{(\beta-\gamma) t} .
$$

On the other hand, dividing Eq. (10) by Eq. (11), one obtains

$$
\frac{\mathrm{d}}{\mathrm{d} n_{r}}\left(n_{i}+n_{r}-1\right)=-\mathrm{R}_{0}\left(n_{i}+n_{r}-1\right),
$$

which immediately yields Eq. (3). Thanks to the explicit dependence $n_{i}\left[n_{r}(t)\right]$, Eq. (11) eventually becomes a first-order nonlinear ODE:

$$
\frac{\mathrm{d} n_{r}}{\mathrm{~d} t}=\gamma\left[1-n_{r}(t)-(1-a) e^{-\mathrm{R}_{0} n_{r}(t)}\right] .
$$

As $t \rightarrow \infty, n_{r}$ tends to the value $n_{r, \infty}$ that makes the right hand side vanish:

$$
n_{r, \infty}=1-(1-a) e^{-\mathrm{R}_{0} n_{r, \infty}} \approx 1-e^{-\mathrm{R}_{0} n_{r, \infty}}
$$

In the large- $\mathrm{R}_{0}$ limit, the second term in the right hand side above is small, so that a recursive resolution can be set up. An excellent approximation is given by

$$
n_{r, \infty} \sim 1-e^{-\mathrm{R}_{0}\left(1-e^{-\mathrm{R}_{0}}\right)},
$$

with an absolute error of less than 0.002 for $\mathrm{R}_{0}>3$. Alternatively, one may express $n_{r, \infty}$ in terms of the tabulated Lambert function [23].

It is immediate to see that Eq. (15) can formally be integrated as

$$
\gamma t=\int_{0}^{n_{r}} \frac{\mathrm{d} n}{1-n-(1-a) e^{-\mathrm{R}_{0} n}} .
$$

This expression has been known and studied for a long time [26]. Note that if if both $\gamma$ and $\beta$ are functions of time, such that their ratio $\mathrm{R}_{0}=\beta / \gamma$ remains constant, then Eq. (18) remains valid, with $\gamma t$ replaced by the effective time $\tau=\int \gamma(t) \mathrm{d} t[22]$. Recently, a thorough analysis of the integrand of Eq. (18) was carried out, yielding upper and lower bounds for the function $t\left(n_{r}\right)$ [23]. In addition, series representations of the integral based either on Taylor expansion of the integrand near the origin or based on its poles in the complex- $n$ plane were given in that work. The first of these approaches effectively amounts to assume that $n$ is small and thus generalizes Kermack and McKendrick approximate solution. However, the formulas in [23] are rather involved, making the inversion of the relation $t\left(n_{r}\right)$ laborious. 


\section{$3 \quad$ Matched asymptotic expansions}

The method consists in deriving separate approximations at the early stage and at the later stage of the outbreak. One the one hand, the outer solution has a characteristic time $\gamma^{-1}$ and describes the decaying phase of the epidemic. On the other hand, the inner solution applies to the growing phase and evolves on a shorter time scale. In the language of asymptotics, the $R_{0} \gg 1$ limit produces a boundary layer problem [28]. The two approximations are constrained by matching conditions at intermediate stages of the outbreak. Thanks to the fact that they have an overlapping range of validity, one can then construct a composite solution, Eq. (1), that is uniformly valid in time.

\subsection{Outer solution}

When $n_{r}=O(1), e^{-\mathrm{R}_{0} n_{r}}$ is exponentially small and can at first be neglected in Eq. (15). We thus have

$$
\frac{\mathrm{d} n_{r}}{\mathrm{~d} t} \sim \gamma\left(1-n_{r}\right), \quad \rightarrow n_{r} \sim n_{r}^{(o)}=1-e^{-\gamma\left(t-t_{o}\right)},
$$

where the superscript $(o)$ refers to "outer" approximation and $t_{o}$ is an arbitrary constant. However, knowing the actual limiting value of $n_{r}$ as $t \rightarrow \infty$, a better approximation is

$$
n_{r}^{(o)}=n_{r, \infty}-e^{-\gamma\left(t-t_{o}\right)} .
$$

Since $n_{r, \infty}$ and 1 only differ by an exponentially small quantity, they are asymptotically equivalent as $\mathrm{R}_{0} \rightarrow \infty$. However, the correction brought about by using $n_{r, \infty}$ in Eq. (20) considerably improves the approximation for moderately large $\mathrm{R}_{0}$ and thus expands the range of usability of the final result, Eq. (1).

Note that $n_{r}^{(o)}$ is only defined for $t>t_{o}$, since $n_{r}$ must be a positive number.

\subsection{Inner solution}

At earlier stages of the epidemics, $n_{r}$ is $O\left(\mathrm{R}_{0}{ }^{-1}\right)$. More precisely, Eq. (13) suggests to write

$$
n \sim n_{r}^{(i)}(t)=\frac{\nu(t)}{\mathrm{R}_{0}-1}
$$

where $(i)$ refers to "inner" approximation. Then Eq. (15) becomes

$$
\begin{aligned}
\frac{\mathrm{d} \nu}{\mathrm{d} t} & =\left(\mathrm{R}_{0}-1\right) \gamma\left[1-e^{-\frac{\mathrm{R}_{0}}{\mathrm{R}_{0}-1} \nu}(1-a)-\frac{1}{\mathrm{R}_{0}-1} \nu\right], \\
& \sim(\beta-\gamma)\left[1-e^{-\nu}(1-a)\right] .
\end{aligned}
$$

Unlike Eq. (15), this last equation can be integrated explicitly:

$$
\frac{e^{\nu}}{e^{\nu}-1+a} \frac{\mathrm{d} \nu}{\mathrm{d} t} \sim \beta-\gamma, \quad \rightarrow \ln \left(\frac{e^{\nu}-1+a}{a}\right) \sim(\beta-\gamma) t,
$$

where we used the fact that $\nu(0)=0$. Hence,

$$
\nu \sim \ln \left[a e^{(\beta-\gamma) t}-1+a\right]
$$

and

$$
n_{r}^{(i)} \sim \frac{1}{\mathrm{R}_{0}-1} \ln \left[a e^{(\beta-\gamma) t}-1+a\right] .
$$




\subsection{Matching}

So far, we have derived two approximations that efficiently describe the solution at different moments of the epidemic. However, the constant $t_{o}$ in Eq. (20) is still unknown. To determine it, we must impose that (20) and (26) match in an intermediate region. Loosely speaking, one wants that

$$
\lim _{t \rightarrow \infty} n_{r}^{(i)}(t) \sim \lim _{t \rightarrow 0} n_{r}^{(o)}(t) .
$$

To formalise this statement more precisely, let us define the matching region as

$$
t=t_{*}+\eta \Delta t
$$

where $\eta$ is such that $\gamma \eta \Delta t \ll 1$, while $(\beta-\gamma) \eta \Delta t \gg 1$. This double asymptotic constraint is possible since $(\beta-\gamma) / \gamma=\mathrm{R}_{0}-1 \gg 1$ by assumption. On the one hand, we have

$$
\begin{aligned}
n_{r}^{(o)}\left(t_{*}+\eta \Delta t\right) & =n_{r, \infty}-e^{-\gamma\left(t_{*}-t_{o}\right)-\gamma \eta \Delta t} \\
& \sim n_{r, \infty}-e^{\gamma\left(t_{*}-t_{o}\right)}+e^{-\gamma\left(t_{*}-t_{o}\right)} \gamma \eta \Delta t .
\end{aligned}
$$

On the other hand, the inner solution yields

$$
\begin{aligned}
n_{r}^{(i)} & \sim \frac{1}{\mathrm{R}_{0}-1} \ln \left[a e^{(\beta-\gamma) t}-1+a\right] \\
& =\frac{1}{\mathrm{R}_{0}-1}\left(\ln \left[a e^{(\beta-\gamma) t}\right]+\ln \left[1-\frac{1-a}{a} e^{-(\beta-\gamma) t}\right]\right) \\
& =\frac{1}{\mathrm{R}_{0}-1}\left\{(\beta-\gamma) t+\ln a+\ln \left[1-\frac{1-a}{a} e^{-(\beta-\gamma) t}\right]\right\} \\
& =\gamma t+\frac{\ln a}{\mathrm{R}_{0}-1}+\frac{1}{\mathrm{R}_{0}-1} \ln \left[1-\frac{1-a}{a} e^{-(\beta-\gamma) t}\right] \\
& \sim \gamma t_{*}+\gamma \eta \Delta t+\frac{\ln a}{\mathrm{R}_{0}-1} .
\end{aligned}
$$

Equating expressions (30) and (35), we obtain the two conditions

$$
n_{r, \infty}-e^{-\gamma\left(t_{*}-t_{o}\right)}=\gamma t_{*}+\frac{\ln a}{\mathrm{R}_{0}-1}, \quad \text { and } \quad e^{-\gamma\left(t_{*}-t_{o}\right)}=1 .
$$

Hence, we have $t_{o}=t_{*}$ and

$$
\gamma t_{*}=n_{r, \infty}-1-\frac{\ln a}{\mathrm{R}_{0}-1} .
$$

\subsection{Composite solution}

As indicated above, $n_{r}^{(o)}$ is only defined for $t>t_{o}=t_{*}$. For $t<t_{*}$, only the inner solution holds:

$$
n_{r}\left(t<t_{*}\right) \sim n_{r}^{(i)} .
$$

On the other hand, for $t>t_{*}$, we may construct a uniformly valid approximation as

$$
n_{r}\left(t>t_{*}\right) \sim n_{r}^{(i)}+n_{r}^{(o)}-(\text { common part }),
$$


where the common part is the time-dependance that $n_{r}^{(i)}$ and $n_{r}^{(o)}$ have in common in the matching region, and which should be removed to avoid double counting. One has, from (35),

$$
(\text { common part })=\gamma t_{*}+\gamma \eta \Delta t+\frac{\ln a}{\mathrm{R}_{0}-1}=\gamma t+\frac{\ln a}{\mathrm{R}_{0}-1} .
$$

Hence

$$
\begin{gathered}
n_{r}\left(t>t_{*}\right) \sim \frac{1}{\mathrm{R}_{0}-1} \ln \left[a e^{(\beta-\gamma) t}-1+a\right]+n_{r, \infty}-e^{-\gamma\left(t-t_{*}\right)}-\gamma t-\frac{\ln a}{\mathrm{R}_{0}-1} \\
=\frac{1}{\mathrm{R}_{0}-1} \ln \left[1-\frac{1-a}{a} e^{-(\beta-\gamma) t}\right]+n_{r, \infty}-e^{-\gamma\left(t-t_{*}\right)}
\end{gathered}
$$

Eqs. (38) and (41) yield Eq. (1), which completes the derivation.

\section{Conclusion}

We have shown that an explicit approximate solution, Eq. (1), can be derived in the limit of large $R_{0}$. The interest of this solution is that it is already effective when the reproduction is only moderately large. Given that SIR is in itself a crude approximation of reality, it makes little sense from an applied perspective to look for an approximation that is exact to within 1\%. Taking the time between inflexion and maximum of the epidemic curve as a measure of accuracy, the present theory, Eq. (9) yields an answer with less than $10 \%$ error for $\mathrm{R}_{0}$ just above 4 . With influenza, a reproduction number exceeding the value of 3 appears rare but possible [29], as was the case with the 1918 influenza pandemic [30]. In the case of the COVID-19 pandemic estimates yield $R_{0}$ in the range 1.5 to 6.49 [31] and particularly around 3 in France [32] and between 3.5 and 4 in Korea and in the Hubei province (China) during the first wave [33]. For measles, $\mathrm{R}_{0}$ can be on the order of 10 or even larger [34]. Hence, the large- $\mathrm{R}_{0}$ limit considered here is relevant, even though, of course, the assumption of a constant ratio $\beta / \gamma$ does not apply to the COVID-19 pandemic. While the formulas derived in this paper appear accurate enough, it is to be noted that they can be improved. Only leading-order expressions have been used for the inner and outer solutions that apply to the ascending and receding phases of the outbreak, respectively. The inner solution can be improved by the keeping $O\left(1 /\left(\mathrm{R}_{0}-1\right)\right)$ corrections in Eq. (22) and treating them as perturbations. The same can be done with $O\left(e^{-\mathrm{R}_{0} n_{r}}\right)$ terms in the equation for the outer solution. Even within the present theory, a correction to $t_{2}$ could be computed by perturbation, thus improving Eq. (9).

\section{Aknowledgement}

G.K. is a Research Associate with the Belgian Fonds de la Recherche Scientifique (FNRS).

\section{A Derivation of formulas (4) and (5)}

From Eq. (10), $\mathrm{d} n_{i} / \mathrm{d} t$ vanishes when $n_{i}=1-\frac{1}{\mathrm{R}_{0}}-n_{r}$. By substitution into (3), one immediately obtains (5). 
Next, let us enquire when the second time derivative of $n_{i}$ vanishes. Writing $n_{i}=n_{i}\left[n_{r}(t)\right]$, let us denote

$$
n_{i}^{\prime}=\frac{\mathrm{d} n_{i}}{\mathrm{~d} n_{r}}
$$

Then,

$$
\begin{aligned}
\frac{\mathrm{d} n_{i}}{\mathrm{~d} t} & =n_{i}^{\prime} \frac{\mathrm{d} n_{r}}{\mathrm{~d} t} \\
\frac{\mathrm{d}^{2} n_{i}}{\mathrm{~d} t^{2}} & =n_{i}^{\prime \prime}\left(\frac{\mathrm{d} n_{r}}{\mathrm{~d} t}\right)^{2}+n_{i}^{\prime} \frac{\mathrm{d}^{2} n_{r}}{\mathrm{~d} t^{2}}=n_{i}^{\prime}\left(\frac{\mathrm{d} n_{r}}{\mathrm{~d} t}\right)^{2}\left[\frac{n_{i}^{\prime \prime}}{n_{i}^{\prime}}+\left(\frac{\mathrm{d} n_{r}}{\mathrm{~d} t}\right)^{-2} \frac{\mathrm{d}^{2} n_{r}}{\mathrm{~d} t^{2}}\right],
\end{aligned}
$$

and one thus seek for the condition

$$
\frac{n_{i}^{\prime \prime}}{n_{i}^{\prime}}=-\left(\frac{\mathrm{d} n_{r}}{\mathrm{~d} t}\right)^{-2} \frac{\mathrm{d}^{2} n_{r}}{\mathrm{~d} t^{2}}
$$

Dividing (10) by (11), one has

$$
n_{i}^{\prime}=\mathrm{R}_{0}-1-\mathrm{R}_{0}\left(n_{i}+n_{r}\right) .
$$

Hence, $n_{i}^{\prime \prime}=-\mathrm{R}_{0}\left(n_{i}^{\prime}+1\right)$ and

$$
\begin{aligned}
\frac{n_{i}^{\prime \prime}}{n_{i}^{\prime}}=-\mathrm{R}_{0}\left(\frac{n_{i}^{\prime}+1}{n_{i}^{\prime}}\right)= & -\mathrm{R}_{0}\left(\frac{\mathrm{R}_{0}-\mathrm{R}_{0}\left(n_{i}+n_{r}\right)}{\mathrm{R}_{0}-1-\mathrm{R}_{0}\left(n_{i}+n_{r}\right)}\right) \\
= & \mathrm{R}_{0} \frac{\mathrm{R}_{0}(1-a) e^{-\mathrm{R}_{0} n_{r}}}{1-\mathrm{R}_{0}(1-a) e^{-\mathrm{R}_{0} n_{r}}} \sim \mathrm{R}_{0} \frac{\mathrm{R}_{0} e^{-\mathrm{R}_{0} n_{r}}}{1-\mathrm{R}_{0} e^{-\mathrm{R}_{0} n_{r}}},
\end{aligned}
$$

as $a \rightarrow 0$. On the other hand, neglecting $a$ compared to 1, (15) yields

$$
\frac{\mathrm{d}^{2} n_{r}}{\mathrm{~d} t^{2}}=-\gamma \frac{\mathrm{d} n_{r}}{\mathrm{~d} t}\left(1-\mathrm{R}_{0} e^{-\mathrm{R}_{0} n_{r}}\right)
$$

Hence,

$$
-\left(\frac{\mathrm{d} n_{r}}{\mathrm{~d} t}\right)^{-2} \frac{\mathrm{d}^{2} n_{r}}{\mathrm{~d} t^{2}}=\gamma\left(\frac{\mathrm{d} n_{r}}{\mathrm{~d} t}\right)^{-1}\left(1-\mathrm{R}_{0} e^{-\mathrm{R}_{0} n_{r}}\right)=\frac{1-\mathrm{R}_{0} e^{-\mathrm{R}_{0} n_{r}}}{1-n_{r}-e^{-\mathrm{R}_{0} n_{r}}} .
$$

Equating (47) with (49) yields (4).

\section{References}

[1] Dilip Kumar Bagal, Arati Rath, Abhishek Barua, and Dulu Patnaik. Estimating the parameters of susceptible-infected-recovered model of COVID19 cases in India during lockdown periods. Chaos, Solitons \& Fractals, 140:110154, nov 2020.

[2] Weston C. Roda, Marie B. Varughese, Donglin Han, and Michael Y. Li. Why is it difficult to accurately predict the COVID-19 epidemic? Infectious Disease Modelling, 5:271-281, 2020.

[3] Giuseppe C. Calafiore, Carlo Novara, and Corrado Possieri. A time-varying SIRD model for the COVID-19 contagion in italy. Annual Reviews in Control, 50:361-372, 2020. 
[4] Ashutosh Simha, R Venkatesha Prasad, and Sujay Narayana. A simple stochastic sir model for COVID-19 infection dynamics for Karnataka: Learning from Europe. arXiv preprint arXiv:2003.11920, 2020.

[5] Miguel Casares and Hashmat Khan. A dynamic model of COVID-19: Contagion and implications of isolation enforcement. Technical report, Carleton Economics Working Papers (CEWP). Carleton University, May 2020.

[6] Yu Chen, Jin Cheng, Yu Jiang, and Keji Liu. A time delay dynamical model for outbreak of 2019-nCoV and the parameter identification. Journal of Inverse and Ill-posed Problems, 28(2):243-250, apr 2020.

[7] Rajiv Chowdhury, , Kevin Heng, Md Shajedur Rahman Shawon, Gabriel Goh, Daisy Okonofua, Carolina Ochoa-Rosales, Valentina GonzalezJaramillo, Abbas Bhuiya, Daniel Reidpath, Shamini Prathapan, Sara Shahzad, Christian L. Althaus, Nathalia Gonzalez-Jaramillo, and Oscar H. Franco. Dynamic interventions to control COVID-19 pandemic: a multivariate prediction modelling study comparing 16 worldwide countries. European Journal of Epidemiology, 35(5):389-399, may 2020.

[8] Marino Gatto, Enrico Bertuzzo, Lorenzo Mari, Stefano Miccoli, Luca Carraro, Renato Casagrandi, and Andrea Rinaldo. Spread and dynamics of the COVID-19 epidemic in Italy: Effects of emergency containment measures. Proceedings of the National Academy of Sciences, 117(19):10484-10491, apr 2020.

[9] Saulo B. Bastos and Daniel O. Cajueiro. Modeling and forecasting the early evolution of the Covid-19 pandemic in Brazil. Scientific Reports, 10(1), nov 2020 .

[10] Giulia Giordano, Franco Blanchini, Raffaele Bruno, Patrizio Colaneri, Alessandro Di Filippo, Angela Di Matteo, and Marta Colaneri. Modelling the COVID-19 epidemic and implementation of population-wide interventions in Italy. Nature Medicine, 26(6):855-860, apr 2020.

[11] Juliane F. Oliveira, Daniel C. P. Jorge, Rafael V. Veiga, Moreno S. Rodrigues, Matheus F. Torquato, Nivea B. da Silva, Rosemeire L. Fiaccone, Luciana L. Cardim, Felipe A. C. Pereira, Caio P. de Castro, Aureliano S. S. Paiva, Alan A. S. Amad, Ernesto A. B. F. Lima, Diego S. Souza, Suani T. R. Pinho, Pablo Ivan P. Ramos, and Roberto F. S. Andrade. Mathematical modeling of COVID-19 in 14.8 million individuals in Bahia, Brazil. Nature Communications, 12(1), jan 2021.

[12] Wout Merbis. Exact epidemic models from a tensor product formulation. arXiv preprint arXiv:2102.11708, 2021.

[13] Fred Brauer, Carlos Castillo-Chavez, and Carlos Castillo-Chavez. Mathematical models in population biology and epidemiology, volume 2. Springer, 2012.

[14] Gregory Kozyreff. Hospitalization dynamics during the first COVID19 pandemic wave: SIR modelling compared to Belgium, France, Italy, Switzerland and New York City data. Infectious Disease Modelling, 6:398404, 2021. 
[15] William Ogilvy Kermack and Anderson G McKendrick. A contribution to the mathematical theory of epidemics. Proc. R. Soc. Lond. A, 115(772):700-721, 1927.

[16] Fred Brauer. The kermack-McKendrick epidemic model revisited. Mathematical Biosciences, 198(2):119-131, dec 2005.

[17] Hina Khan, Ram N. Mohapatra, K. Vajravelu, and S.J. Liao. The explicit series solution of SIR and SIS epidemic models. Applied Mathematics and Computation, 215(2):653-669, sep 2009.

[18] Tiberiu Harko, Francisco S.N. Lobo, and M.K. Mak. Exact analytical solutions of the Susceptible-Infected-Recovered (SIR) epidemic model and of the SIR model with equal death and birth rates. Applied Mathematics and Computation, 236:184-194, 2014.

[19] Martin Bohner, Sabrina Streipert, and Delfim F.M. Torres. Exact solution to a dynamic SIR model. Nonlinear Analysis: Hybrid Systems, 32:228-238, may 2019 .

[20] Nathaniel S. Barlow and Steven J. Weinstein. Accurate closed-form solution of the SIR epidemic model. Physica D: Nonlinear Phenomena, 408:132540, jul 2020 .

[21] Riccardo Giubilei. Closed form solution of the SIR model for the COVID-19 outbreak in italy. medRxiv, jun 2020.

[22] M Kröger and R Schlickeiser. Analytical solution of the SIR-model for the temporal evolution of epidemics. part a: time-independent reproduction factor. Journal of Physics A: Mathematical and Theoretical, 53(50):505601, nov 2020

[23] Piet Van Mieghem. Exact solution of the Kermack and McKendrick SIR differential equations. arXiv:2010.03253, 2020.

[24] Fernando Menezes Campello de Souza. An approximate analytical solution to the SIR model. Available at SSRN: https://ssrn.com/abstract=3780708 or http://dx.doi.org/10.2139/ssrn.3780708, 2021.

[25] Nikolay A. Kudryashov, Mikhail A. Chmykhov, and Michael Vigdorowitsch. Analytical features of the SIR model and their applications to COVID-19. Applied Mathematical Modelling, 90:466-473, feb 2021.

[26] David G Kendall. Deterministic and stochastic epidemics in closed populations. In Proc. 3rd Berkeley Symp. Math. Statist. Prob, volume 4, pages 149-165, 1956.

[27] David E. Clark, Gavin Welch, and Jordan S. Peck. Approximate relationships between SIR and logistic models. medRxiv, 2020.

[28] Carl M. Bender and Steven A. Orszag. Advanced Mathematical Methods for Scientists and Engineers: Asymptotic Methods and Perturbation Theory ( $v$. 1). Springer, 1999. 
medRxiv preprint doi: https://doi.org/10.1101/2021.03.24.21254226; this version posted March 29, 2021. The copyright holder for this preprint (which was not certified by peer review) is the author/funder, who has granted medRxiv a license to display the preprint in perpetuity.

It is made available under a CC-BY 4.0 International license .

[29] Laura Forsberg White, Jacco Wallinga, Lyn Finelli, Carrie Reed, Steven Riley, Marc Lipsitch, and Marcello Pagano. Estimation of the reproductive number and the serial interval in early phase of the 2009 influenza A/H1N1 pandemic in the USA. Influenza and Other Respiratory Viruses, 3(6):267276 , nov 2009.

[30] Christina E. Mills, James M. Robins, and Marc Lipsitch. Transmissibility of 1918 pandemic influenza. Nature, 432(7019):904-906, dec 2004.

[31] Ying Liu, Albert A Gayle, Annelies Wilder-Smith, and Joacim Rocklv. The reproductive number of COVID-19 is higher compared to SARS coronavirus. Journal of Travel Medicine, 27(2), feb 2020.

[32] Sarah A. Nadeau, Timothy G. Vaughan, Jérémie Scire, Jana S. Huisman, and Tanja Stadler. The origin and early spread of SARS-CoV-2 in Europe. Proceedings of the National Academy of Sciences, 118(9):e2012008118, feb 2021.

[33] Sunhwa Choi and Moran Ki. Estimating the reproductive number and the outbreak size of COVID-19 in Korea. Epidemiology and Health, 42:e2020011, mar 2020.

[34] Fiona M Guerra, Shelly Bolotin, Gillian Lim, Jane Heffernan, Shelley L Deeks, Ye Li, and Natasha S Crowcroft. The basic reproduction number $\left(\mathrm{R}_{0}\right)$ of measles: a systematic review. The Lancet Infectious Diseases, 17(12):e420-e428, dec 2017. 